\title{
Deposition of Cellulose Nanocrystals onto Supported Lipid Membranes
}

Yotam Navon, Bruno Jean, Liliane Coche-Guérente, Franck Dahlem, Anne BernheimGroswasser, *and Laurent Heux*

Supporting Information
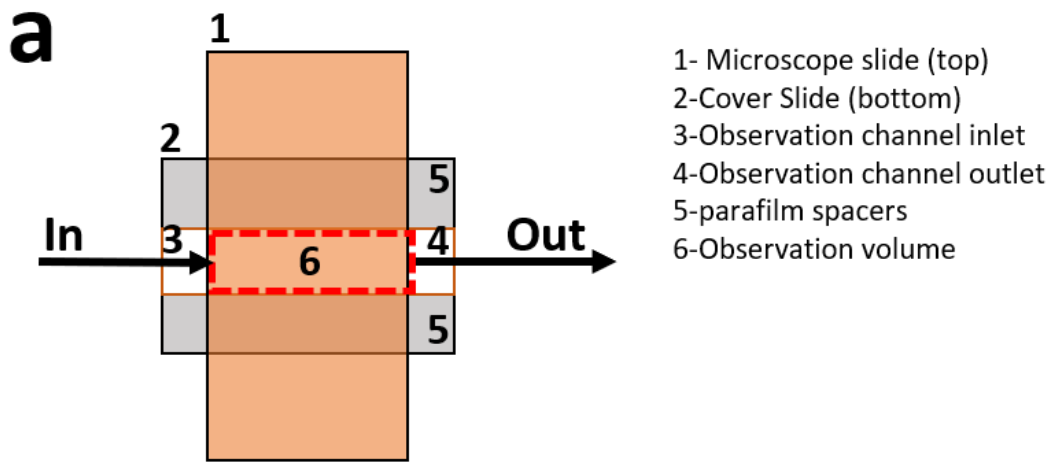

b

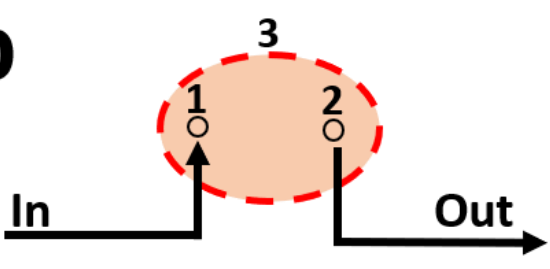

1- Measurement chamber inlet

2- Measurement chamber outlet

3-Crystal surface

Figure SI1. The measurement configuration in (a) the TIRF observation cell and (b) the QCMD module. Arrows indicate the direction of flow in the probed area, which was $1 \mathrm{~cm}^{2}$ with total volume of $50 \mu \mathrm{L}$ in both cases. 


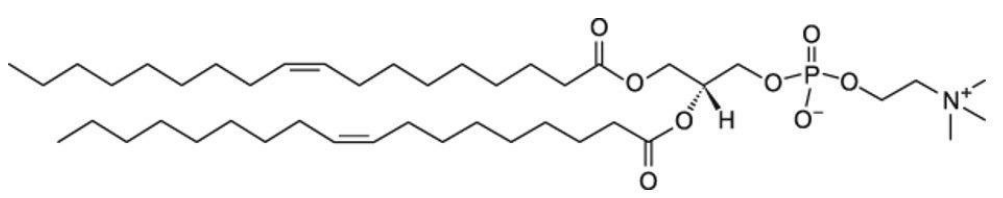

1,2-dioleoyl-sn-glycero-3-phosphatidylcholine (DOPC)<smiles>CCCCCCCC/C=C\CCCCCCCCCCCCC(=O)OCC(C[N+](C)(C)[ClH+])OC(=O)CCCCCCCCCC</smiles>

1,2-dioleoyl-3 trimethylammonium-propane (DOTAP)

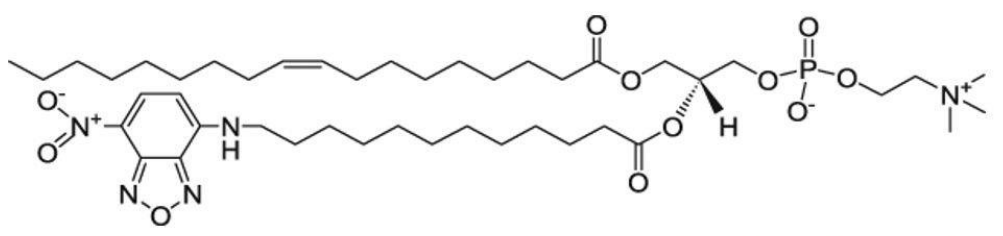

1-palmitoyl-2-(6-[(7-nitro-2-1,3-benzoxadiazol-4-yl)amino] hexanoyl)-sn-glycero-3-phosphocholine (NBD-PC)

Figure SI2. Chemical structure of the lipids used to create the SLBs.
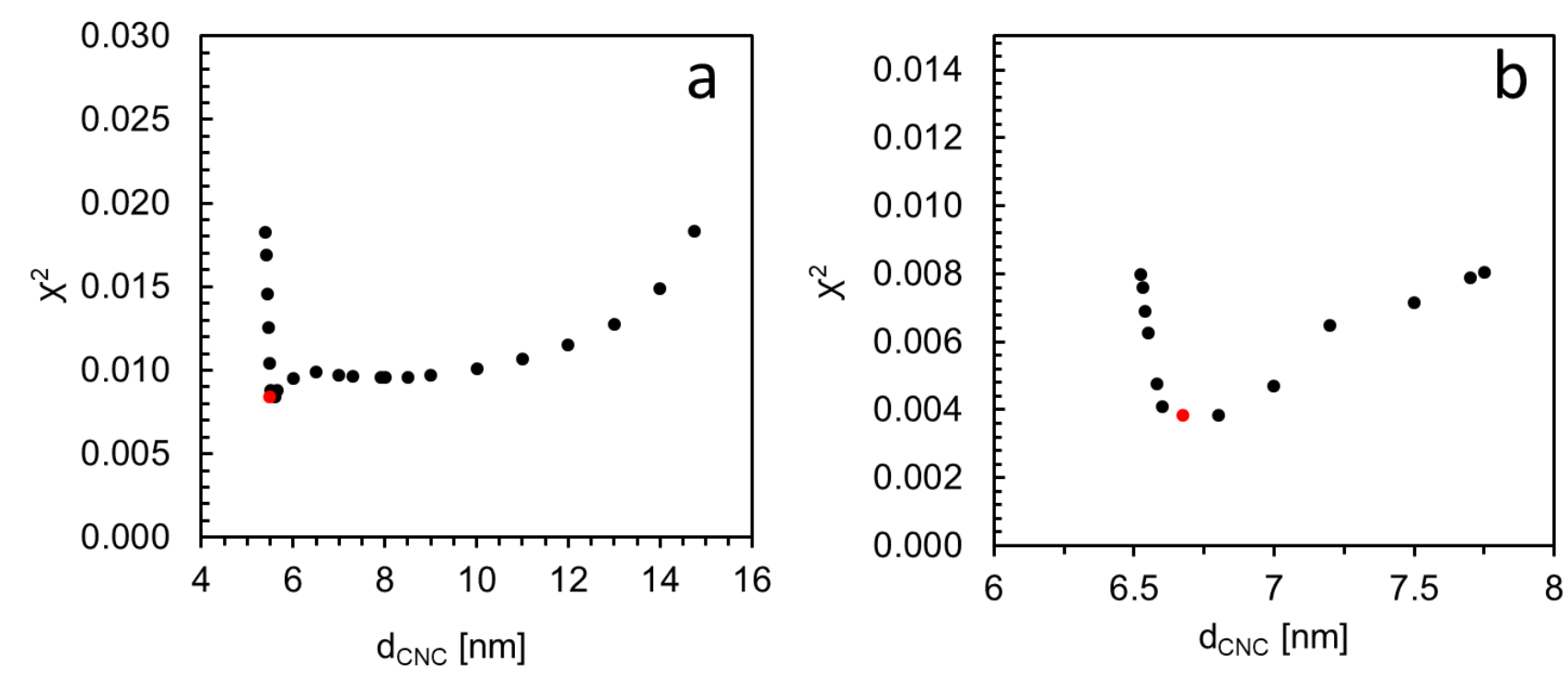

Figure SI3. $\chi^{2}$ landscape for the CNC layer deposited on DOPC/DOTAP (a) and DOPC (b) systems. The red point indicates the minimal solution and the extreme points represent the solution boundaries for a confidence interval of 0.683 or one standard deviation. 
$(\mathrm{A})$

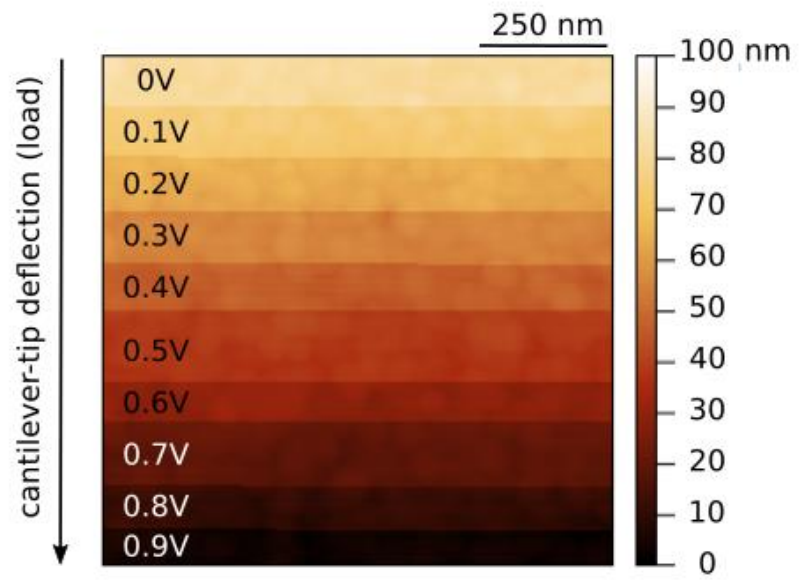

(B)

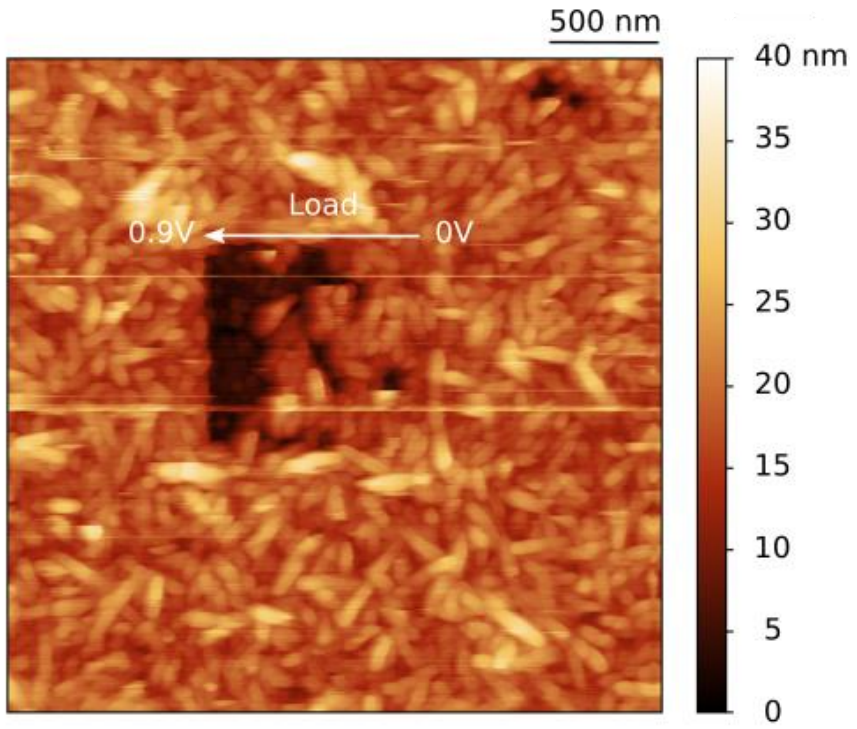

FigureSI4. Protocol for the nanoshaving procedure: (A) SFM topography image in contact mode made of 256 scanning lines and by changing every 25 lines the force load expressed as cantilevertip deflection in Volt. The z-change is due to the modification of the piezotube position to obtain the required cantilever deflection. The granular surface of the substrate is visible even at the lowest deflection. The lateral size of the grains increases with the load due to the deterioration of the tip radius. The scan direction was selected to be at 90 degrees from the main cantilever axis in order to record a proper lateral signal. This one changes with a few hundred of milliVolts. (B) $3 \times 3 \mu \mathrm{m}^{2}$ topography image done at the zero angle with a PeakForce setpoint of $600 \mathrm{pN}$. Three zones are distinguished. Between 0 and $0.3 \mathrm{~V}$, the construct displays a minor deformation. Starting around $0.3 \mathrm{~V}$, few $\mathrm{CNC}$ were removed and above $0.7 \mathrm{~V}$ deflection the whole film was peeled off. 


\section{SFM Data processing via Gwyddion.}

A data leveling was first done inside the groove by fitting a plane through three points (Fig. SI5 A). These points were positioned at the top of the grain substrate. The height of each point was averaged over 16 pixels (i.e. $48 \mathrm{~nm}$ ). After a plane removing, four extracted profile lines at different angles and averaged over 3 pixels were plotted to control the plane subtraction (Fig. SI5 B). All of these lines do not present slope and their values are in the range of $4 \mathrm{~nm}$ corresponding to the substrate roughness. The flatness control was done on the $\mathrm{CNC}$ part with an averaging over 128 lines, in order to avoid error related to the large surface roughness (Fig. SI5 C). Lines profiles show more dispersion but stay flat, the step height can be extracted. This was done in both orientations (Fig. SI5 D), giving the same step value.
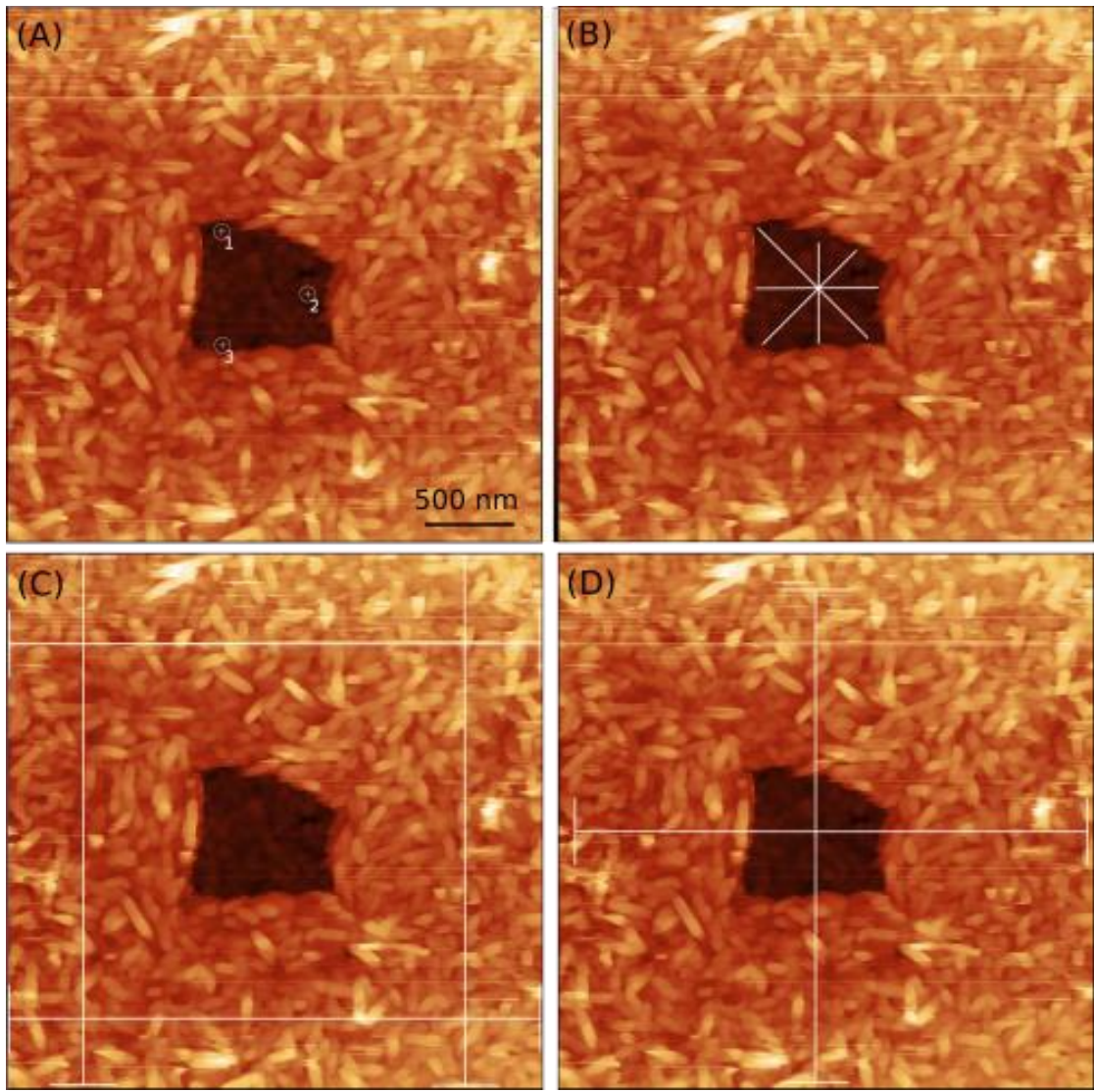
Figure SI5. (A) Inside the groove, three points averaged over 16 pixels were used to fit a plan and level data. (B) After plane removing, four extract profile lines at different angles and averaged over 3 pixels were plotted in order to control the previous plane subtraction. (C) Flatness control was also done on the CNC surface with 128 averaged lines. (D) Final result of the step height was confirmed on both orientations. 